\title{
TAILS FROM THE ORPHANAGE
}

\author{
CARl J. Grillmair \\ IPAC, Mail Code 314-6, California Institute of Technology, 1200 E. California Blvd., Pasadena, CA 91125, USA; carl@ipac.caltech.edu \\ Received 2016 November 4; revised 2016 November 18; accepted 2016 November 22; published 2017 January 5
}

\begin{abstract}
Examining a portion of the northern Sloan Digital Sky Survey (SDSS) footprint, we detect at least three and possibly seven halo debris streams. One of these (PS1-D) was recently detected in the Pan-STARRS1 $3 \pi$ survey, and the remaining two are also evident as extensions of the SDSS detections. All of these streams are metal-poor and are found at a distance of around $21 \pm 5 \mathrm{kpc}$. The streams are between $65^{\circ}$ and $70^{\circ}$ in length, oriented almost north-south, and are nearly parallel and somewhat convergent with the neighboring Orphan stream. Surface densities ranging from 1.5 to 0.5 stars per square degree down to $g=21.7$ correspond to surface brightnesses between 35 and 37 mag per square arcsecond. The streams each appear to be more than $300 \mathrm{pc}$ across, suggesting either dwarf/ultrafaint galaxy progenitors or long-term heating of very ancient globular cluster streams. The orbits of all but one of these streams appear to be nearly radial, and the orbit normals suggest that all of the streams are part of the Vast Polar Structure, a relatively narrow plane that contains most of the known satellite galaxies, globular clusters, and stellar streams.
\end{abstract}

Key words: Galaxy: halo - Galaxy: structure

\section{INTRODUCTION}

Dozens of distinct, highly collimated stellar debris streams are now known to orbit in the halo of our Galaxy (see Grillmair \& Carlin 2016 and Smith 2016 for reviews). Detecting and tracing such streams around the Galaxy will become increasingly important as we refine our techniques for using them as probes of Galactic potential (Küpper et al. 2015; Bovy et al. 2016) and of the distribution of dark matter subhalos (Carlberg 2009; Yoon et al. 2011). Knowing in advance the locations and trajectories of these streams may also help us interpret the vast amount of data we expect to harvest from upcoming Gaia data releases.

While searching for and examining other streams in the Sloan Digital Sky Survey (SDSS), we have long noted a somewhat fibrous texture in the area of sky surrounding the Orphan stream (the "orphanage"). However, detailed examinations at a high spatial resolution $(0.1)$ have not revealed any obvious streams at a signal-to-noise ratio $(\mathrm{S} / \mathrm{N})$ sufficient for publication. In this Paper we coarsen our spatial sampling considerably, enabling the detection of at least three nearly parallel, low-metallicity stellar debris streams. We describe our detection method in Section 2. We make an initial attempt to constrain the orbits in Section 3 and discuss additional lowsignificance structures in Section 4. Concluding remarks are given in Section 5.

\section{ANALYSIS}

We use the photometric catalog from data release 10 of the SDSS. Experience has shown that we probe most deeply and are most sensitive to differences in stellar populations using just the $g, r$, and $i$ measurements. We use all objects classed as stars and with $g<21.7$. Photometry is dereddened using the DIRBE/IRAS dust maps of Schlegel et al. (1998), corrected using the prescription of Schlafly \& Finkbeiner (2011).

Figure 1 shows the result of applying a matched filter in the color-magnitude domain to the western half of the northern footprint of the SDSS. The filter is based on the color-magnitude distribution of stars in the old, metal-poor globular cluster
NGC 5053 with $[\mathrm{Fe} / \mathrm{H}] \approx-2.29$ (Harris 1996). Three streams become apparent, running roughly north-south and somewhat convergent with the Orphan stream, similar in appearance to the claw marks that bears commonly leave on trees.

Figure 2 shows the distribution of $E(B-V)$ over the same region of sky as in Figure 1 (Schlegel et al. 1998). While there are evidently some random clumps of dust emission between the streams, there are no linear features that resemble the streams in Figure 1. We conclude that the features in Figure 1 are not an artifact of reddening-induced incompleteness.

Figure 3 shows the same part of the sky in a matched-filtered map of the Pan-STARRS1 $3 \pi$ survey (Bernard et al. 2016) for a distance of $25 \mathrm{kpc}$. While there are evidently issues with spatially variable completeness at the limit of the single-epoch survey, each of the streams in Figure 1 has a counterpart in Figure 3 extending to the southern limit of the Pan-STARRS survey. That the streams are not as obvious as they are in Figure 1 is presumably at least partly due to the more metalrich $([\mathrm{Fe} / \mathrm{H}]=-1.5)$ isochrone used by Bernard et al. (2016). For example, the Orphan stream appears considerably weaker in Figure 3 than it does in Grillmair (2006a), Belokurov et al. (2006), or Newberg et al. (2010).

The features in Figures 1 and 3 extend through the constellations Leo, Leo Minor, Hydra, Sextans, Antlia, and Pyxis. Since there are also multiple streams in the same constellations, we follow Grillmair (2014) and name the streams after rivers cited in the Illiad. Henceforth we refer to the stream next to PS1-D as Sangarius, and the stream next to Orphan as Scamander.

The trajectories of the streams north of the bright, southern Sagittarius arm in panel d of Figure 1are obviously somewhat conjectural. While they appear plausible, the discontinuity created by the Sagittarius stream creates some ambiguity, and velocity information will be required before we can definitively conclude that the northern extensions are not unrelated structures. Including both northern (Figure 1) and southern portions (Figure 3 ) of the streams, the arc lengths are $68^{\circ}, 59^{\circ}$, and $66^{\circ}$ for PS1-D, Sangarius, and Scamander, respectively. At a distance of $21 \mathrm{kpc}$, this translates to physical lengths of 25 , 22 , and $24 \mathrm{kpc}$, respectively. 


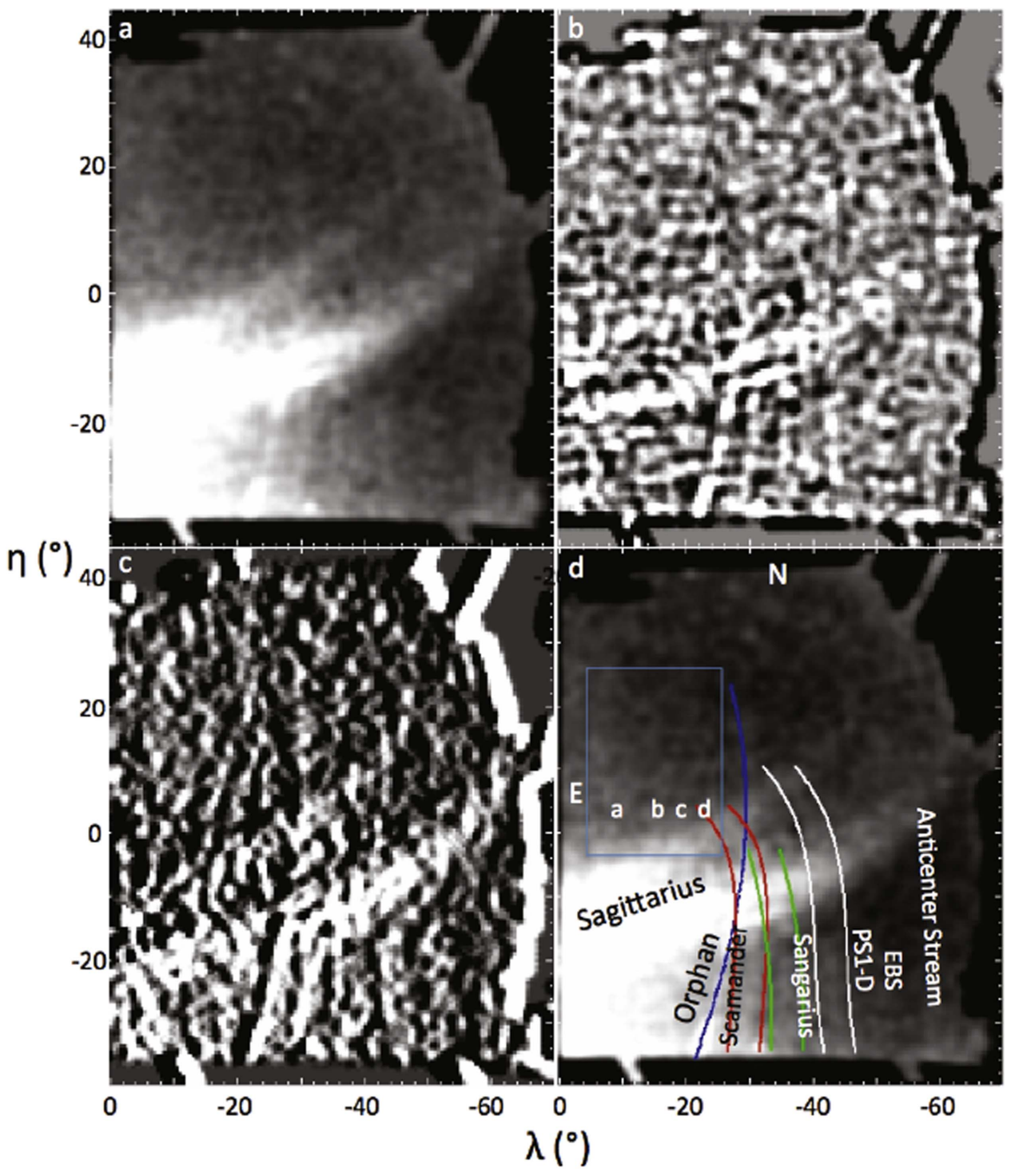

Figure 1. Panel (a): filtered surface density map of the $70^{\circ} \times 85^{\circ}$ western portion of the northern SDSS footprint, in SDSS coordinates. The SDSS coordinate system has the advantage that small differences in calibration and completeness from scan to scan are aligned east-west, or very nearly along horizontal lines in the figure. The matched filter is based on the color-magnitude distribution of stars in NGC 5053 , shifted to a distance of $21 \mathrm{kpc}$. The map has been binned to $0.5 \times 0.5$ pixels and smoothed with a Gaussian kernel of 1.5. The stretch is linear, with lighter areas indicating higher surface densities. Panel (b): the same filtered image as in panel (a) after subtraction of a smoothed background image constructed using an annular median window filter of radius 4.5. Panel (c): the map in panel (a) after subtracting a version of itself that is shifted $1^{\circ}$ to the east. Panel (d): same as panel (a), but with the streams indicated. The curves correspond to Equations ((1))-(3), shifted east and west by 2.5 . The blue curve shows the Orphan stream trajectory of Newberg et al. (2010). The light blue box indicates the location of four possible streams (labeled a, b, c, and d) discussed in Section 4.

In equatorial coordinates, the trajectory of PS1-D can be modeled to $\sigma=0.19$ using:

$$
\begin{aligned}
\alpha= & 141.017+0.208 \delta-0.02491 \delta^{2}+0.000609 \delta^{3} \\
& -1.20989 \times 10^{-6} \delta^{4} .
\end{aligned}
$$

Sangarius is well modeled $(\sigma=0.22)$, with:

$$
\alpha=148.9492-0.03811 \delta+0.001505 \delta^{2},
$$

while Scamander again requires a higher-order fit $\left(\sigma=00^{\circ} 11\right)$ :

$$
\begin{aligned}
\alpha= & 155.642-0.1000 \delta-0.00191 \delta^{2}-0.0003346 \delta^{3} \\
& +1.47775 \times 10^{-5} \delta^{4} .
\end{aligned}
$$

Grillmair (2009) quantified the significance of streams above the background using the " $T$ " statistic, measuring the median filtered surface density in multiple segments as one sweeps across the sky perpendicular to the orientation of the stream. The region of sky in the vicinity of the orphanage is quite complex, with the Sagittarius, Orphan, EBS, and Anticenter streams, along with the orphanage streams themselves, making it difficult to measure the noise floor in a stream-free region. In Figure 4 we show the run of $T$ across an unsmoothed version of Figure 1, normalizing to the field rms measured in an identical manner in an apparently blank region of sky to the north and east of the Sagittarius stream. We use Equations (1)-(3) to define the stream segments we pass over each respective stream. We detect PS1-D and Scamander at roughly the $5 \sigma$ level, while we detect Sangarius at somewhere between 2 and $4 \sigma$. These levels roughly accord with the visual impression in Figure 1. 


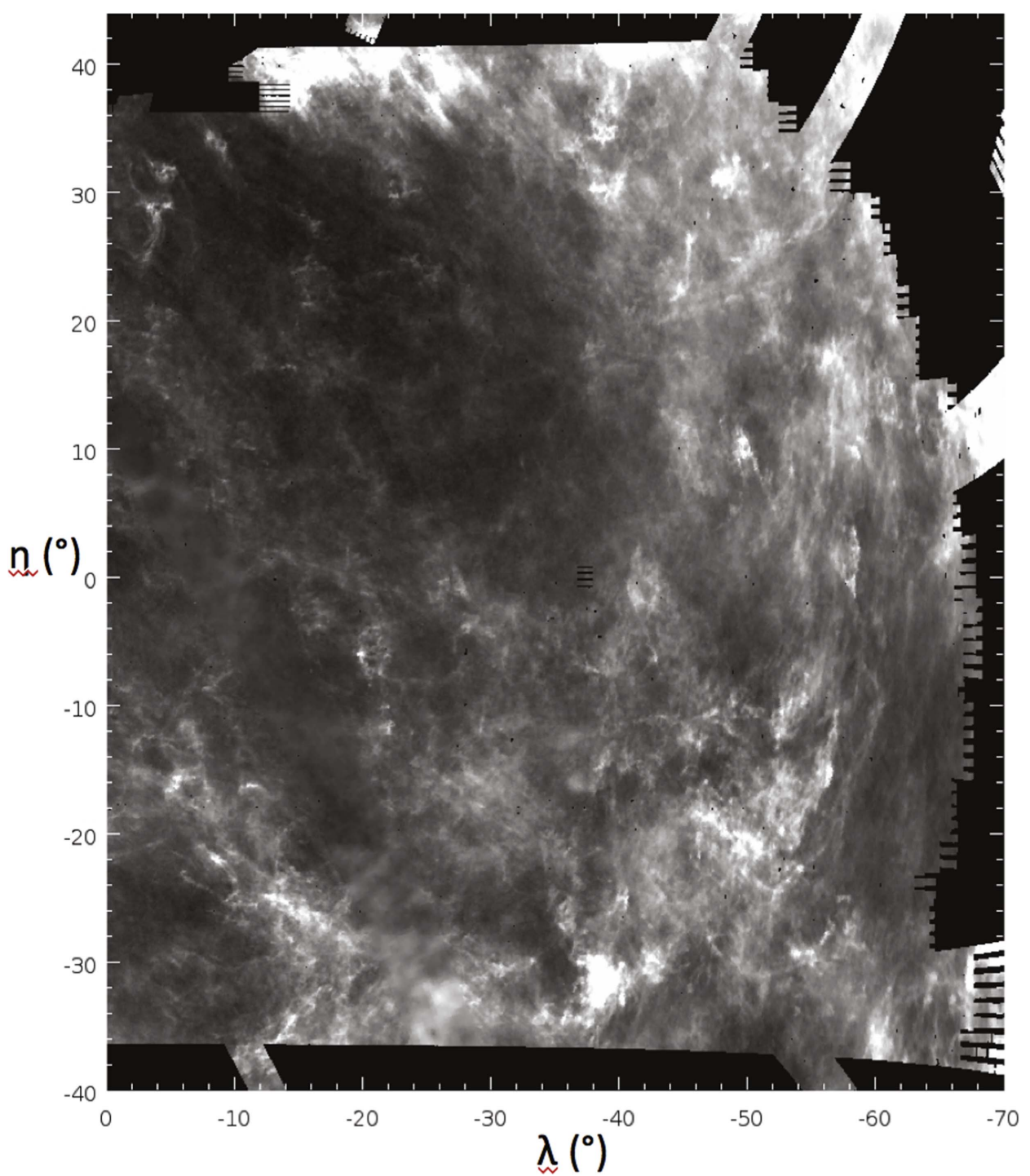

Figure 2. The distribution of $E(B-V)$, taken from Schlegel et al. (1998), in the same coordinate system as Figure 1. The stretch is linear, with white indicating $E(B-V)>0.09$ and black corresponding to $E(B-V)<0.01$.

Sampling a version of Figure 1 using 0.1 binning, we find that the FWHMs of the streams range from 0.9 to 1.1 . At a distance of $21 \mathrm{kpc}$, this corresponds to physical widths ranging from 330 to $400 \mathrm{pc}$, broader than most of the known globular cluster streams but narrower than the Orphan stream. This suggests that the streams could be either the remnants of diminutive dwarf or ultrafaint galaxies, or (more likely given our population estimates below) globular cluster streams that have been heated for $10 \mathrm{Gyr}$ or more by dark-matter subhalos (Carlberg 2009).

Color-magnitude diagrams for the streams are shown in Figure 5. All of the streams appear to be quite metal-poor ([Fe/ $\mathrm{H}] \leqslant-1.4)$, which is expected since our filter was designed to capture metal-poor substructures. To estimate the number of stars in each stream, we count only stars that lie within $2 \sigma$ of the NGC 5053 color-magnitude locus and on the subgiant branch or below. Once again, given the complexity of the field, it is difficult to measure the surface density of field stars. Our best estimate yields mean stream surface densities of 0.5 stars per square degree for the southernmost $15^{\circ}\left(-35^{\circ}<\eta<-20^{\circ}\right)$ of PS1-D and Sangarius in Figure 1 and 1.5 stars per square degree for the same portion of Scamander. These stream surface densities are superposed on a field star surface density, selected using the same photometric constraints, of about 10 stars per square degree. We are therefore sampling the streams with a total of between 30 and 110 stars down to $g=21.7$. If we adopt a globular cluster-like luminosity function and integrate down the main sequence, then for a distance of $21 \mathrm{kpc}$, we find that there should be between 440 (Sangarius) and 1600 (Scamander) stars in total within these portions of the streams. A similar integration yields equivalent surface brightnesses of between 35 and $37 \mathrm{mag}$ per square arcsecond. If the stream segments we see are portions of streams that encircle the Galaxy, then we arrive at total populations of the progenitors of between $10^{4}$ and $4 \times 10^{4}$ stars, e.g., comparable to the populations of modern-day globular clusters.

There are no known globular clusters or dwarf galaxies falling near great circles defined by each stream, though this is not conclusive as the uncertainties are high. However, the widths of the streams, combined with their tenuousness, may be an indication of great age and of the possibility that the progenitors dissolved long ago. 


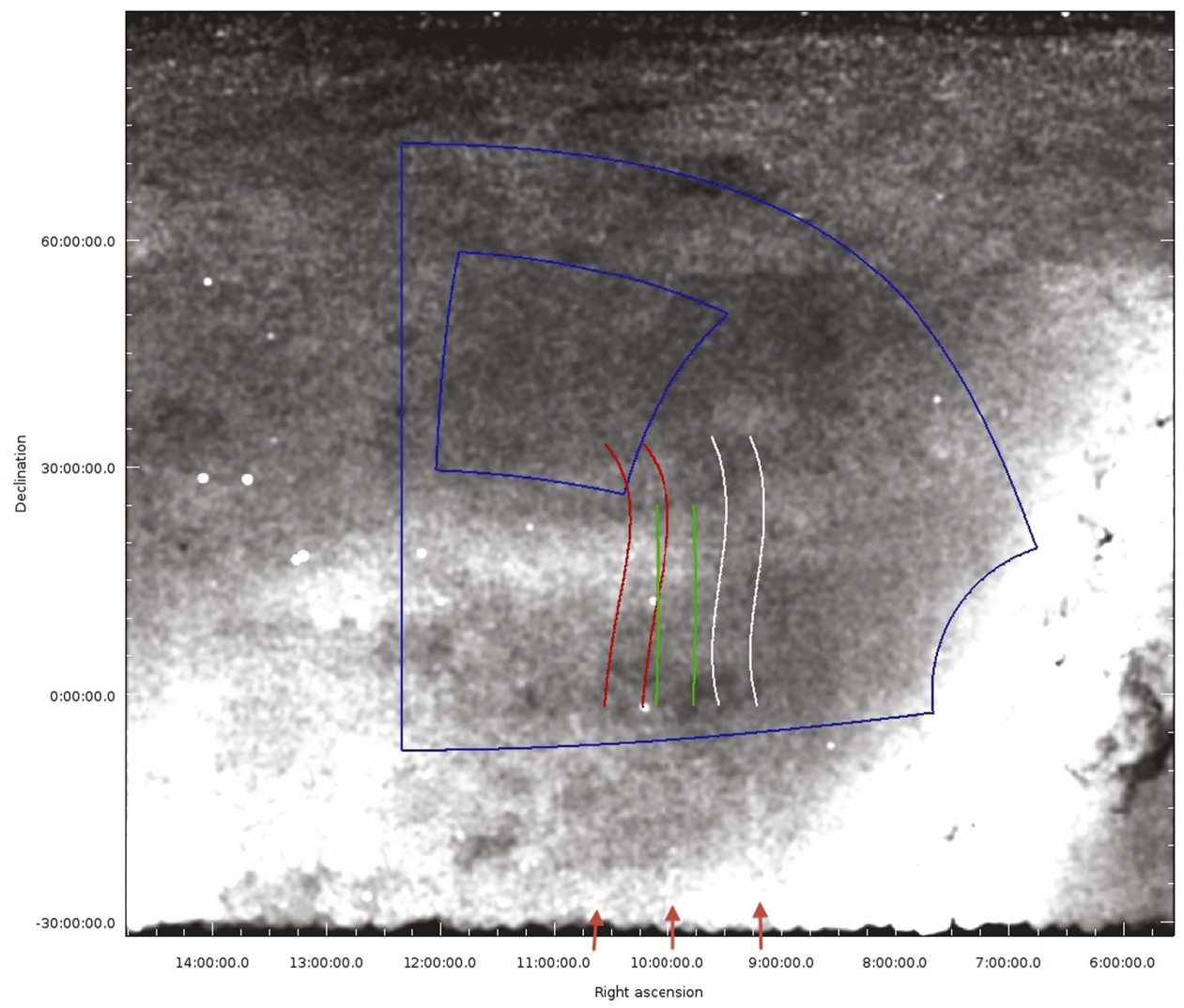

Figure 3. A portion of Bernard et al.'s (2016) matched-filtered density map of stars in a single epoch of the Pan-STARRS $3 \pi$ survey. In this case, the stars have been filtered using an isochrone with an age of $12 \mathrm{Gyr}$, a metallicity of $[\mathrm{Fe} / \mathrm{H}]=-1.5$, and a distance of $25 \mathrm{kpc}$. The blue boxes indicate the region shown in Figure 1, and the red, green, and white curves are the same as those in panel d of Figure 1. The stretch is linear, with lighter areas indicating higher surface densities. Despite the significant pattern noise due to variations in depth and completeness, Sangarius, Scamander, and PS1-D all show indications of continuing southward, as indicated by the arrows.

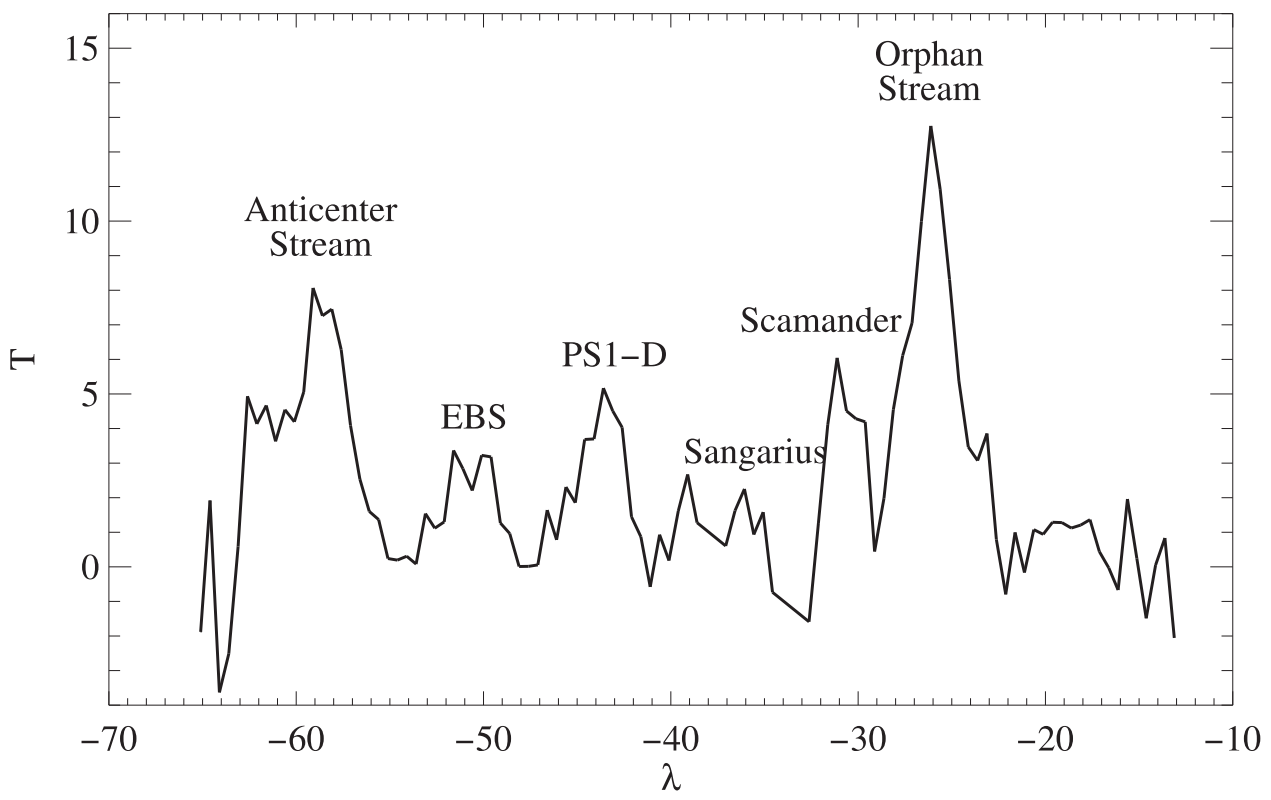

Figure 4. The " $T$ " statistic of Grillmair (2009) for the southern $15^{\circ}$ of the streams in Figure 1. $T$ is the median value, measured from the image filtered for a distance of $21 \mathrm{kpc}$, for five $3^{\circ}$ long segments in each stream. We have normalized the run of $T$ by dividing by the rms measured in the same way in an apparently blank region of sky north of the Sagittarius stream. The plotted values thus correspond roughly to the $\mathrm{S} / \mathrm{N}$ at each point. The peaks corresponding to the Anticenter (Grillmair 2006b; Li et al. 2012), EBS (Grillmair 2011; Hargis et al. 2016), and Orphan streams (Grillmair 2006a; Belokurov et al. 2007; Newberg et al. 2010; Sesar et al. 2013; Grillmair et al. 2015) show up only incidentally and are significantly lower than the S/Ns we would measure using more appropriate filters, correct distances, and suitably oriented stream segments. 

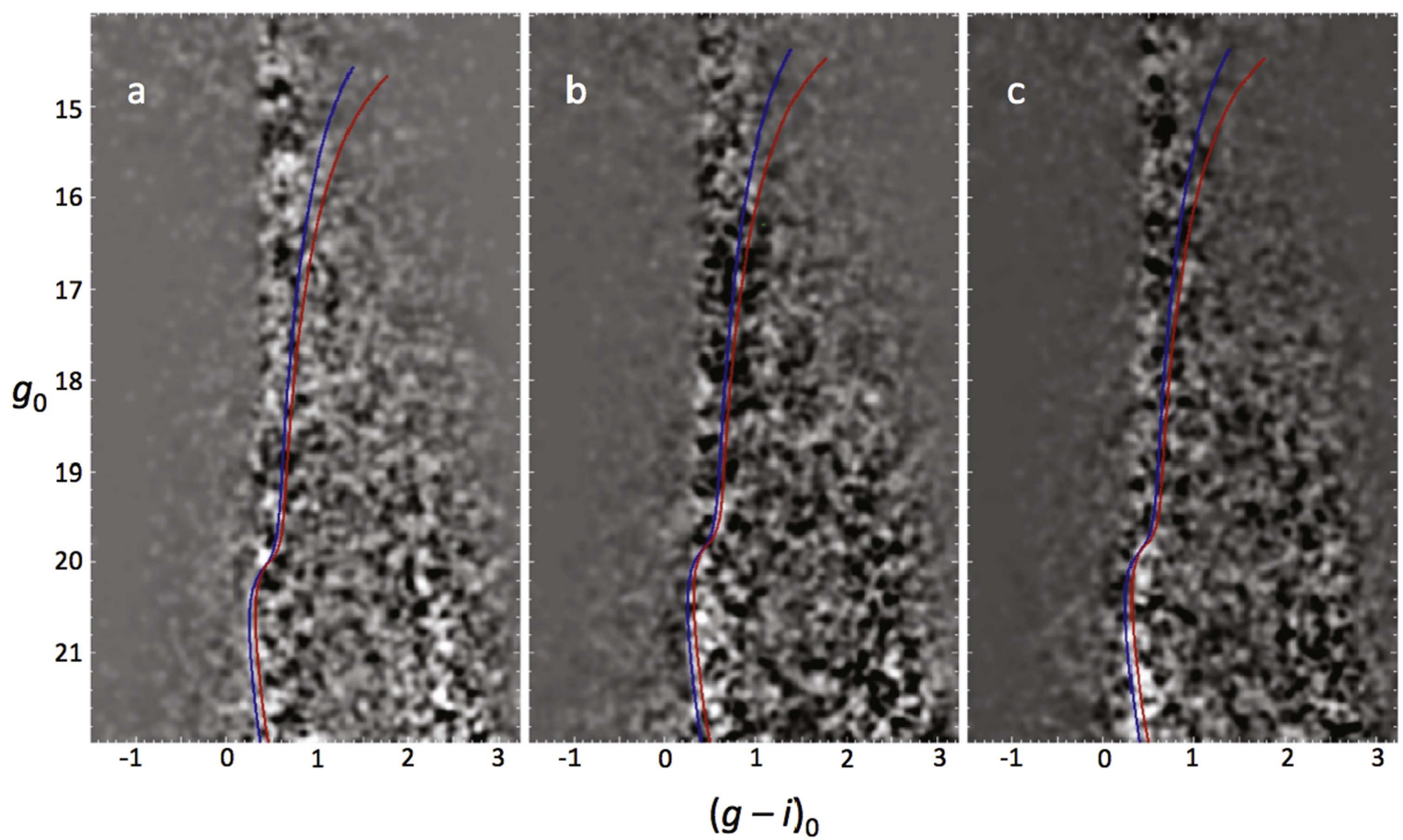

Figure 5. Dereddened $(g-i)_{0}$ Hess diagrams for (a) PS1-D, (b) Sangarius, and (c) Scamander. The curves show the theoretical loci for $Z=0.0001$ ([Fe/H] $=-2.2$ ) in blue and for $Z=0.0007([\mathrm{Fe} / \mathrm{H}]=-1.44)$ in red.

Table 1

Predicted Motions and Orbit Parameters

\begin{tabular}{|c|c|c|c|c|}
\hline & & PS1-D & Sangarius & Scamander \\
\hline \multirow[t]{2}{*}{ Fiducial Point } & R.A. $\left({ }^{\circ}, \mathrm{J} 2000\right)$ & 141.457 & 149.013 & 153.520 \\
\hline & decl. $\left({ }^{\circ}, \mathrm{J} 2000\right)$ & +11.301 & +7.847 & +12.460 \\
\hline \multirow{3}{*}{ Prograde Orbit } & $v_{\text {hel }}\left(\mathrm{km} \mathrm{s}^{-1}\right)$ & $63 \pm 109$ & $156_{-421}^{+314}$ & $151_{-46}^{+38}$ \\
\hline & $\mu_{\alpha} \cos (\delta)\left(\operatorname{mas~yr}^{-1}\right)$ & $-0.31 \pm 0.06$ & $-0.78 \pm 0.19$ & $-0.47 \pm 0.02$ \\
\hline & $\mu_{\delta}\left(\operatorname{mas~yr}^{-1}\right)$ & $+3.16 \pm 0.9$ & $+6.21_{-1.0}^{+2.0}$ & $-1.01 \pm 0.07$ \\
\hline \multirow{3}{*}{ Retrograde Orbit } & $v_{\text {hel }}\left(\mathrm{km} \mathrm{s}^{-1}\right)$ & $159 \pm 109$ & $84_{-314}^{+421}$ & $53_{-38}^{+46}$ \\
\hline & $\mu_{\alpha} \cos (\delta)\left(\operatorname{mas~yr}^{-1}\right)$ & $+0.14 \pm 0.06$ & $+0.21 \pm 0.19$ & $-0.25 \pm 0.02$ \\
\hline & $\mu_{\delta}\left(\operatorname{mas} \mathrm{yr}^{-1}\right)$ & $-7.0 \pm 0.9$ & $-9.89_{-1.0}^{+2.0}$ & $-2.55 \pm 0.07$ \\
\hline$R_{\text {apo }}(\mathrm{kpc})$ & $\cdots$ & $>100$ & $>100$ & $29 \pm 2$ \\
\hline$R_{\text {peri }}(\mathrm{kpc})$ & $\cdots$ & $27 \pm 0.3$ & $25 \pm 0.2$ & $6 \pm 1$ \\
\hline$i\left(^{\circ}\right)$ & $\cdots$ & $64 \pm 3$ & $79 \pm 5$ & $34 \pm 1$ \\
\hline$\epsilon$ & $\cdots$ & $>0.6 \pm 0.3$ & $>0.6 \pm 0.3$ & $0.66 \pm 0.03$ \\
\hline \multirow[t]{2}{*}{ Orbit Pole } & $l\left({ }^{\circ}\right)$ & $183 \pm 2$ & $178 \pm 5$ & $196 \pm 2$ \\
\hline & $b\left({ }^{\circ}\right)$ & $-57 \pm 1$ & $-52 \pm 1$ & $-41 \pm 2$ \\
\hline
\end{tabular}

\section{ORBITS}

Though we have as yet no velocity information for these streams, and while we are mindful that streams do not precisely follow single orbits (Eyre \& Binney 2011), the distances and trajectories of the streams can give us some idea of the orbit parameters. We use a model for the Galactic potential by Allen \& Santillan (1991) and fit in a least-squares sense to five or six roughly equidistant normal points along each stream in the region $-35^{\circ}<\eta<0^{\circ}$. We assign a distance of $21 \mathrm{kpc}$ to the middle and the ends of each stream. While our distance estimates are far less certain than the stream trajectories on the sky, we find that the ends of the streams disappear if we shift the selection filter by more than $\approx 0.5 \mathrm{mag}$ faintward or brightward; the streams are evidently nearly perpendicular to our line of sight. We assign 0.3 uncertainties to the positions of the individual normal points and assign $5 \mathrm{kpc}$ uncertainties to our distance estimates.

Table 1 lists the orbit parameters corresponding to the best fits. The uncertainties are estimated by setting each of the three free parameters (the radial velocity and the two components of proper motion) to their $90 \%$ limits and refitting the stream using the remaining two parameters. While the orbital planes are quite well constrained by the observed trajectories of the streams, the radial velocities and hence eccentricities are evidently nearly unconstrained.

The convex-eastward curvature found by Bernard et al. (2016) for PS1-D is at odds with any plausible orbit around the 
Galaxy. This westward-bending feature at the north end of the stream is visible in panels (b) and (c) of Figure 1, though in the SDSS data it is neither an obvious continuation of the stream nor clearly preferred over the eastward-bending feature we trace in panel (d). The stream trace in panel (d) alleviates the nonphysical aspect of the orbit, though only just. This suggests that the northern part of the Bernard et al. (2016) stream may actually be some unrelated structure. Deeper photometric data and/or Gaia proper motions will be required to determine the true path of PS1-D north of the Sagittarius stream.

Both our eastward-bending PS1-D and Sangarius appear to be on nearly radial orbits, while Scamander is on a less extreme, somewhat more tightly bound orbit. We note that the Orphan stream is also on a far-flung orbit, with $R_{\text {apo }} \simeq 90 \mathrm{kpc}$ (Newberg et al. 2010). This suggests the possibility that PS1-D and Sangarius may be dynamically related to Orphan in some way, perhaps through a much larger structure that gave rise to Orphan. Table 1 shows that the orbital poles for PS1-D, Sangarius, and Scamander all lie between those of the Orphan and Anticenter streams. This consequently puts them within the region defining the Vast Polar Structure (VPOS) (Pawlowski et al. 2012) that apparently contains the orbits of most of the known dwarf galaxies, globular clusters, and stellar debris streams orbiting our Galaxy.

\section{STILL OTHER STREAMS?}

Careful examination of Figure 1 shows that there may be at least four more streams, at S/Ns considerably lower than those of the streams analyzed above, at a distance of $\approx 21 \mathrm{kpc}$ in the region $0^{\circ}>\lambda>-25^{\circ},-5^{\circ}<\eta<25^{\circ}$. These ladder-like structures are almost certainly affected by discontinuities in the SDSS scan direction, but the enhancements in the northsouth direction are less easy to dismiss.

If indeed these are additional streams, then their similar orientations suggest that either the data or our filtering and analysis are somehow particularly sensitive to streams oriented in a nearly north-south direction, or that the VPOS once contained many more objects (clusters or dwarf galaxies) than we see today. Concerning the former, we are certainly biased against selecting structures oriented along the SDSS east-west scan direction, but we know of no process or peculiarity that could enhance apparently linear structures in the cross-scan direction. These potential streams will need to be verified once we have access to other surveys (e.g., Pan-STARRS) and other stream detection methods (e.g., Gaia). We mention them here primarily to aid in the identification or verification of structures that may be discovered in upcoming Gaia releases.

These features appear to have trajectories qualitatively similar to those of the three streams examined above. Orbit fits yield nearly radial orbits, with orbit normals $\left(l, b=199^{\circ} \pm 1^{\circ}, 26^{\circ} \pm 4^{\circ}\right)$ that once again lie within the patch of sky associated with the VPOS.

\section{CONCLUSIONS}

Examining a region of the northern footprint of the SDSS, we find evidence for at least three and possibly seven stellar debris streams. With 30 to 110 stars per stream and equivalent surface brightnesses of $>35 \mathrm{mag} \mathrm{arcsec}^{-2}$, these streams are likely at the very limit of what can be detected in the SDSS. Yet they are enticing as perhaps the tip of a substantial iceberg, as well as an indicator of what we may discover when Gaia proper motions become available.

All of these streams appear to orbit within the VPOS, believed to contain the majority of surviving satellite galaxies. Once this has been verified with velocity information, these streams will clearly add to the significance of this structure and to the consequences it may have for our understanding of galaxy formation in $\Lambda \mathrm{CDM}$.

We are grateful to Edouard Bernard for making available matched-filtered maps of the Pan-STARRS $3 \pi$ survey. We are also grateful to an anonymous referee for several thoughtful suggestions that improved both the content and readability of the paper.

Facilities: Sloan, PS1.

\section{REFERENCES}

Allen, C., \& Santillan, A. 1991, RMxAA, 22, 255

Belokurov, V., Evans, N. W., Irwin, M. J., et al. 2007, ApJ, 658, 337

Belokurov, V., Zucker, D. B., Evans, N. W., et al. 2006, ApJL, 642, L137

Bernard, E. J., Ferguson, A. M. N., Schlafly, E. F., et al. 2016, MNRAS, 463, 1759

Bovy, J., Bahmanyar, A., Fritz, T. K., et al. 2016, arXiv:1609.01298 Carlberg, R. G. 2009, ApJ, 705, 223

Eyre, A., \& Binney, J. 2011, MNRAS, 413, 1852

Grillmair, C. J. 2006a, ApJL, 645, L37

Grillmair, C. J. 2006b, ApJL, 651, L29

Grillmair, C. J. 2009, ApJ, 693, 1118

Grillmair, C. J. 2011, ApJ, 738, 98

Grillmair, C. J. 2014, ApJ, 790, 10

Grillmair, C. J., \& Carlin, J. L. 2016, in Tidal Streams in the Local Group and Beyond, ed. H. J. Newberg \& J. L. Carlin (Berlin: Springer), 87

Grillmair, C. J., Hetherington, L., Carlberg, R. G., \& Willman, B. 2015, ApJL, 812, L26

Hargis, J. R., Kimmig, B., Willman, B., et al. 2016, ApJ, 818, 39

Harris, W. E. 1996, AJ, 112, 1487

Küpper, A. H. W., Balbinot, E., Bonaca, A., et al. 2015, ApJ, 803, 80

Li, J., Newberg, H. J., \& Carlin, J. L. 2012, ApJ, 757, 151

Newberg, H. J., Willett, B. A., Yanny, B., \& Xu, Y. 2010, ApJ, 711, 32

Pawlowski, M. S., Pflamm-Altenburg, J., \& Kroupa, P. 2012, MNRAS, 423, 1109

Schlafly, E. F., \& Finkbeiner, D. P. 2011, ApJ, 737, 103

Schlegel, D. J., Finkbeiner, D. P., \& Davis, M. 1998, ApJ, 500, 525

Sesar, B., Grillmair, C. J., Cohen, J. G., et al. 2013, ApJ, 776, 26

Smith, M. C. 2016, in Tidal Streams in the Local Group and Beyond, ed. H. J. Newberg \& J. L. Carlin (Berlin: Springer), 113

Yoon, J. H., Johnston, K. V., \& Hogg, D. W. 2011, ApJ, 731, 58 